\title{
The impact of PPARa activation on whole genome gene expression in human precision cut liver slices
}

\author{
Aafke W.F. Janssen ${ }^{1}$, Bark Betzel ${ }^{2}$, Geert Stoopen ${ }^{3}$, Frits J. Berends², Ignace M. Janssen², Ad A. Peijnenburg ${ }^{3}$ \\ and Sander Kersten ${ }^{1 *}$
}

\begin{abstract}
Background: Studies in mice have shown that PPARa is an important regulator of lipid metabolism in liver and key transcription factor involved in the adaptive response to fasting. However, much less is known about the role of PPARa in human liver.

Methods: Here we set out to study the function of PPARa in human liver via analysis of whole genome gene regulation in human liver slices treated with the PPARa agonist Wy14643.

Results: Quantitative PCR indicated that PPARa is well expressed in human liver and human liver slices and that the classical PPARa targets PLIN2, VLDLR, ANGPTL4, CPT1A and PDK4 are robustly induced by PPARa activation. Transcriptomics analysis indicated that 617 genes were upregulated and 665 genes were downregulated by PPARa activation ( $q$ value $<0.05$ ). Many genes induced by PPARa activation were involved in lipid metabolism (ACSL5, AGPAT9, FADS1, SLC27A4), xenobiotic metabolism (POR, ABCC2, CYP3A5) or the unfolded protein response, whereas most of the downregulated genes were involved in immune-related pathways. Among the most highly repressed genes upon PPARa activation were several chemokines (e.g. CXCL9-11, CCL8, CX3CL1, CXCL6), interferon $\gamma$-induced genes (e.g. IFITM1, IFIT1, IFIT2, IFIT3) and numerous other immune-related genes (e.g. TLR3, NOS2, and LCN2). Comparative analysis of gene regulation by Wy 14643 between human liver slices and primary human hepatocytes showed that down-regulation of gene expression by PPARa is much better captured by liver slices as compared to primary hepatocytes. In particular, PPARa activation markedly suppressed immunity/ inflammation-related genes in human liver slices but not in primary hepatocytes. Finally, several putative new target genes of PPARa were identified that were commonly induced by PPARa activation in the two human liver model systems, including TSKU, RHOF, CA12 and VSIG10L.
\end{abstract}

Conclusion: Our paper demonstrates the suitability and superiority of human liver slices over primary hepatocytes for studying the functional role of PPARa in human liver. Our data underscore the major role of PPARa in regulation of hepatic lipid and xenobiotic metabolism in human liver and reveal a marked immuno-suppressive/ anti-inflammatory effect of PPARa in human liver slices that may be therapeutically relevant for non-alcoholic fatty liver disease.

Keywords: Precision cut liver slices, PPARa, Human liver, Transcriptomics

\footnotetext{
* Correspondence: sander.kersten@wur.nl

${ }^{1}$ Nutrition, Metabolism and Genomics Group, Wageningen University,

Stippeneng 4, 6708 WE, Wageningen, The Netherlands

Full list of author information is available at the end of the article
} 


\section{Background}

The Peroxisome Proliferator Activated Receptors (PPARs) represent an important group of receptors involved in mediating the pleiotropic effects of various environmental contaminants, food components, and drugs [1]. PPARs are members of the nuclear receptor superfamily and induce the expression of numerous genes by functioning as ligand-activated transcription factors. The ligands for PPARs encompass a range of synthetic compounds and endogenous lipids, including various fatty acids and eicosanoids. Three different PPAR subtypes can be distinguished: PPAR $\alpha$, PPAR $\delta$, and PPAR $\gamma$, each characterized by a distinct tissue expression profile and set of functions $[2,3]$. Multiple functions have been assigned to PPAR $\delta$, including roles in inflammation, lipid metabolism and cancer [4]. Due to its ubiquitous expression pattern and diverse cellular actions, no single descriptor appropriately captures the biological function of PPAR $\delta$. The PPAR $\gamma$ is known as the key transcriptional regulator that drives adipogenesis [5], the process by which fat cells differentiate from pre-adipocytes into mature adipose cells. Apart from adipocytes, PPAR $\gamma$ is also expressed in a limited number of other cell types where it exerts antiinflammatory actions and promotes lipid storage [6]. By serving as the molecular target of the insulin-sensitizing drugs pioglitazone and rosiglitazone, PPAR $\gamma$ is one of the key receptors in the pharmacological treatment of type 2 diabetes [7].

PPAR $\alpha$ is best known for its role in the liver, where it acts as the master regulator of lipid metabolism, especially during fasting [8-10]. Fasting is associated with dramatic changes in lipid handling in the liver, which is coordinated by PPAR $\alpha$. Specifically, low and high throughput gene expression analyses have demonstrated that PPAR $\alpha$ governs expression of numerous genes involved in nearly every single aspect of lipid metabolism, including fatty acid uptake, mitochondrial and peroxisomal fatty acid oxidation, ketogenesis, and formation and breakdown of triglycerides and lipid droplets [11].

Similar to other members of the PPAR family, PPAR $\alpha$ is activated by a range of different fatty acids and eicosanoids [12-16]. In addition, PPAR $\alpha$ serves as receptor for a diverse array of synthetic compounds collectively referred to as peroxisome proliferators [17]. These include phthalates, insecticides, herbicides, surfactants, organic solvents, and hypolipidemic fibrate drugs. Fibrates have been used for several decades mainly for their ability to lower circulating triglycerides [18]. More recently, pharmacological targeting of PPAR $\alpha$ has shown promise for the treatment of non-alcoholic fatty liver disease. Specifically, the dual PPAR $\alpha / \delta$ agonist GFT505 was shown to improve liver dysfunction markers, decrease hepatic lipid accumulation, and reduce inflammatory gene expression in liver in several animal models of non-alcoholic fatty liver disease
(NAFLD) [19]. Furthermore, GFT505 treatment lowered liver dysfunction markers and improved hepatic and peripheral insulin sensitivity in human subjects $[19,20]$.

Most of our insights into the physiological, toxicological and pharmacological role of PPAR $\alpha$ has been derived from experiments in animals and in particular from rodent studies. These studies have revealed that PPAR $\alpha$ is not only involved in the adaptive response to fasting but also mediates the hepatocarcinogenic effects of peroxisome proliferators [21]. Whether PPAR $\alpha$ exerts similar functions in human liver has been the subject of controversy [22], which has been fueled by the perceived lack of effect of PPAR $\alpha$ agonists on peroxisomal fatty acid oxidation in humans [23], as well as due to the presumed low expression of PPAR $\alpha$ in human liver [24]. However, more recent studies have partly refuted those notions, showing that a) PPAR $\alpha$ expression is similar in mouse and human liver, b) in human hepatocytes PPAR $\alpha$ governs the expression of numerous genes in various lipid metabolic pathways, including peroxisomal fatty acid oxidation $[25,26]$.

Nevertheless, the absence of more complex human model systems has hampered our ability to gain insight into the molecular function of PPAR $\alpha$ in human liver, in particular with respect to target gene regulation. To overcome this limitation, we collected precision cut liver slices (PCLS) from human subjects and studied the effect of PPAR $\alpha$ activation on gene expression using whole genome expression profiling. In contrast to primary hepatocytes, PCLS mimic the multi-cellularity and structural organization of whole liver and thus represent a superior ex-vivo model system for human liver [27]. So far the use of PCLS for the study of nuclear receptors and specifically PPAR $\alpha$ function has been limited [28-30]. Here we report the use of PCLS in combination with whole genome gene expression profiling to gain insight into PPAR $\alpha$-mediated gene regulation in human liver.

\section{Methods}

\section{Collection of liver biopsies}

In all patients a laparoscopic Roux-en-Y Gastric Bypass was performed under general anesthesia as treatment for their morbid obesity. Patients were instructed to fasten for solid foods and liquids starting at the night before surgery. During surgery a biopsy of the liver was obtained with the help of ultrasound dissection (UltraCision ${ }^{\circledR}$ ). The biopsy was collected from the liver edge. The majority of the liver biopsies collected $(n=15)$ were immediately frozen in liquid nitrogen and stored at $-80{ }^{\circ} \mathrm{C}$. The liver biopsies targeted for slicing $(n=4)$ were immediately placed in ice-cold oxygenated Belzer UW Cold Storage Solution (Bridge to Life Ltd, Columbia, SC, USA) and quickly transferred to our laboratory for further processing of PCLS. Only macroscopically healthy livers were used for 
slicing. Biopsies were provided by the biobank of the Rijnstate hospital. Collection of biopsies for research purposes into the biobank was approved by the local institutional review board on behalf of the medical ethics review committee of the Radboud University Medical Centre. All patients signed informed consent for collection of the biopsy prior to surgery. Donor characteristics of the livers used for slicing are shown in Table 1.

\section{Preparation and treatment of precision cut liver slices}

PCLS were prepared and cultured as described previously [31]. $5 \mathrm{~mm}$ cylindrical liver cores were prepared with a surgical biopsy punch and sectioned to $200 \mu \mathrm{m}$ slices using a Krumdieck tissue slicer (Alabama Research and Development, Munford, AL, USA) filled with carbonated KHB ( $\mathrm{pH} 7.4$, supplemented with $25 \mathrm{mM}$ glucose). Liver slices were incubated in William's E Medium (Gibco, Paisley, Scotland) supplemented with penicillin/streptomycin in 6-well plates at $37{ }^{\circ} \mathrm{C} / 5 \% \mathrm{CO}_{2} / 80 \% \mathrm{O}_{2}$ under continuous shaking $(70 \mathrm{rpm})$. Duplicate wells were used per donor with 3 liver slices per well. After 1 hour the media was replaced with fresh William's E Medium in the presence or absence of Wy14643 $(100 \mu \mathrm{M})$ dissolved in dimethyl sulfoxide (DMSO, final concentration $0.1 \%)$. This concentration was chosen based on the affinity of human PPAR $\alpha$ for Wy14643 [32]. After $24 \mathrm{~h}$ incubation, liver slices were snap-frozen in liquid nitrogen and stored in $-80{ }^{\circ} \mathrm{C}$ for RNA isolation.

\section{Primary human hepatocytes}

The treatment of primary human hepatocytes with Wy14643 has been previously described [26]. Briefly, human hepatocytes from six different donors were purchased from Lonza (Verviers, Belgium). Hepatocytes were isolated with two-step collagenase perfusion method. Cell viability was over $80 \%$. The cells were incubated for $24 \mathrm{~h}$ in the presence or absence of Wy14643 $(50 \mu \mathrm{M})$ dissolved in DMSO, followed by RNA isolation.

\section{RNA isolation and qPCR}

Total RNA was isolated using TRIzol reagent (Invitrogen). RNA integrity number was found to be above 7.1 for all samples suggesting that the human liver slices were of good quality. The RNA integrity number is based on a digital electropherogram generated using a Agilent bioanalyzer. It describes the degree of degradation of RNA

Table 1 Donor characteristics of livers used for slicing

\begin{tabular}{llll}
\hline Patient & Gender & Age (years) & Body Mass Index (g/m2) \\
\hline 1 & Female & 34 & 41 \\
2 & Female & 46 & 43 \\
3 & Female & 39 & 35 \\
4 & Female & 41 & 38 \\
\hline
\end{tabular}

with level 10 representing completely intact RNA. RNA was reverse transcribed using a iScript cDNA Synthesis Kit (Bio-Rad Laboratories BV, Veenendaal, The Netherlands). Real-time PCR was carried out using SensiMiX (Bioline) on a CFX 384 Bio-Rad thermal cycler (Bio-Rad). 36B4 was used as housekeeping gene. Primer sequences used are shown in Table 2.

\section{Microarray analysis}

Total RNA was purified with RNeasy Minikit columns (Qiagen) and RNA quality was assessed using RNA 6000 Nano chips on the Agilent 2100 Bioanalyzer (Agilent Technologies, Amsterdam, The Netherlands). Purified RNA (100 ng) was labeled with the Ambion WT expression kit (Invitrogen) and hybridized to an Affymetrix Human Gene 1.1 ST array plate (Affymetrix, Santa Clara, CA). Hybridization, washing, and scanning were carried out on an Affymetrix GeneTitan platform according to the instruction by the manufacturer. Arrays were normalized using the Robust Multiarray Average method $[33,34]$. Probe sets were defined according to Dai et al. [35]. In this method probes are assigned to Entrez IDs as an unique gene identifier. The $P$-value for the effect of Wy14643 treatment were calculated using an Intensity-Based Moderated T-statistic (IBMT) [36]. The $q$-value was calculated as measure of significance for false discovery rate [37]. The microarray data for the human liver slices were submitted to Gene Expression Omnibus (GSE71731). The microarray data for the human primary hepatocytes have been previously submitted to Gene Expression Omnibus (GSE17251).

Gene set enrichment analysis (GSEA) was used to find enriched gene sets in the induced or suppressed genes [38]. Genes were ranked based on the paired IBMTstatistic and subsequently analyzed for over- or underrepresentation in predefined gene sets derived from Gene Ontology, KEGG, National Cancer Institute, PFAM, Biocarta, Reactome and WikiPathways pathway databases. Only gene sets consisting of more than 15 and fewer than 500 genes were taken into account. Statistical significance of GSEA results was determined using 1000 permutations.

Table 2 Primer sequences used for qPCR

\begin{tabular}{lll}
\hline & Primer Sequence & \\
\cline { 2 - 3 } Name & Forward & Reverse \\
\hline 36 44 & CGGGAAGGCTGTGGTGCTG & GTGAACACAAAGCCCACATTCC \\
ANGPTL4 & CACAGCCTGCAGACACAACTC & GGAGGCCAAACTGGCTITGC \\
PLIN2 & ATGGCATCCGTTGCAGTTGA & GATGGTCTTCACACCGTTCTC \\
PDK4 & TGGAGCATTTCTCGCGCTAC & ACAGGCAATTCTTGTCGCAAA \\
CPT1A & TCCAGTTGGCTTATCGTGGTG & CTAACGAGGGGTCGATCTTGG \\
VLDLR & GGTGAAAATGATTGTGACAGTGG & GTGAACTCGTCGGGACTACA \\
\hline
\end{tabular}




\section{Results}

First, we determined the relative expression level of PPAR $\alpha$ in PCLS in comparison with human liver. After correcting for the housekeeping gene 36B4, mRNA levels of PPAR $\alpha$ in human PCLS after $24 \mathrm{~h}$ incubation were about ten-fold lower as compared to snap-frozen human liver biopsies (Fig. 1a). To verify that the human liver slices maintain their ability to respond to PPAR $\alpha$ activation, we exposed the PCLS to $100 \mu \mathrm{M}$ Wy14643, isolated total RNA and performed qPCR to determine the expression of a number of well-established PPAR $\alpha$ target genes, including PLIN2, VLDLR, ANGPTL4, CPT1A and PDK4 (Fig. 1b). All PPAR $\alpha$ target genes analyzed showed significant induction following Wy14643 treatment, indicating the validity of our model to study PPAR $\alpha$-mediated gene regulation.

To study the effect of PPAR $\alpha$ activation on whole genome gene expression, we performed microarray analysis. Wy14643-induced changes in expression of the selected PPAR $\alpha$ target genes were very similar between the

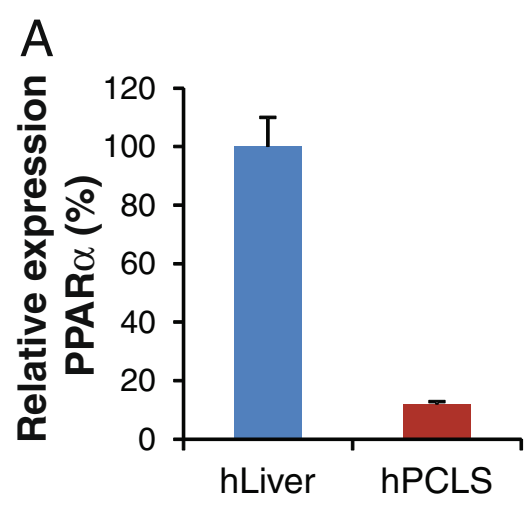

$\mathrm{B}$

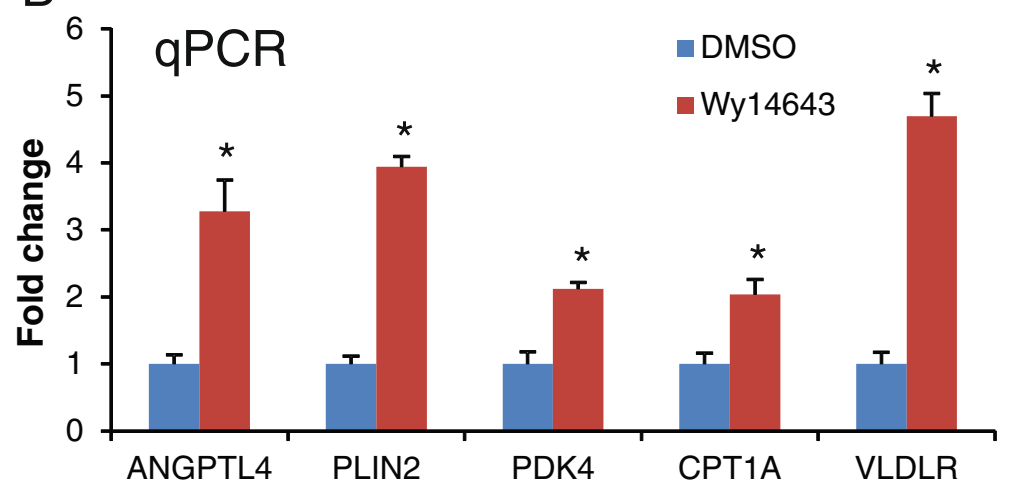

C

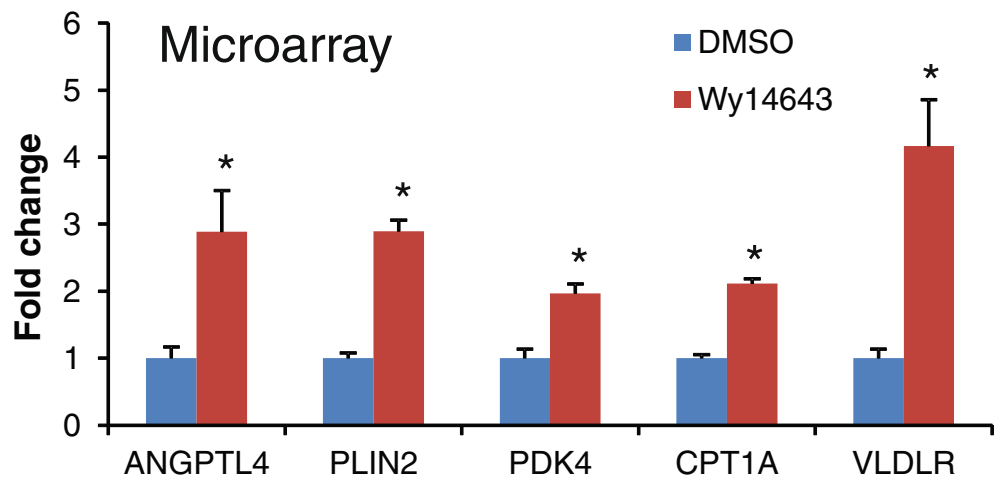

Fig. 1 Classical PPARa targets genes are robustly induced by PPARa activation in human PCLS. a Expression level of PPARa in human liver biopsies $(n=15)$ and human PCLS $(n=5)$. b Gene expression changes of selected PPARa target genes in human PCLS in response to 24 h Wy14643 treatment as determined by quantitative real-time PCR. c Gene expression changes of the same genes according to microarray. Error bars represent SEM. Asterisk indicates statistically significant $(P<0.05$, Student's t-test) 
microarray and qPCR analysis (Fig. 1c). Using a FDR $q$ value of 0.05 as cut-off (IBMT regularised paired $t$-test), the expression of 1282 genes (out of 19,654 genes on the array) was found to be significantly altered by Wy14643 treatment, of which 617 genes were upregulated and 665 genes were downregulated. The top 25 of most significantly induced genes, ranked according to statistical significance, are shown in Fig. 2a. The full list is available as Additional file 1 . The list includes many well-known PPAR $\alpha$ target genes involved in lipid metabolism (e.g.
VLDLR, ACADVL, PLIN2, ANGPTL4, CPT1A), as well as many other genes covering a wide variety of biological functions. In addition, the list includes a number of genes with unknown function. Figure $2 \mathrm{~b}$ shows the top 25 of most significantly repressed genes, many of which are related to immune function and inflammation. The full list of significantly downregulated genes is available as Additional file 2 .

To gain better insight into the biological function of genes regulated by PPAR $\alpha$ activation in human liver

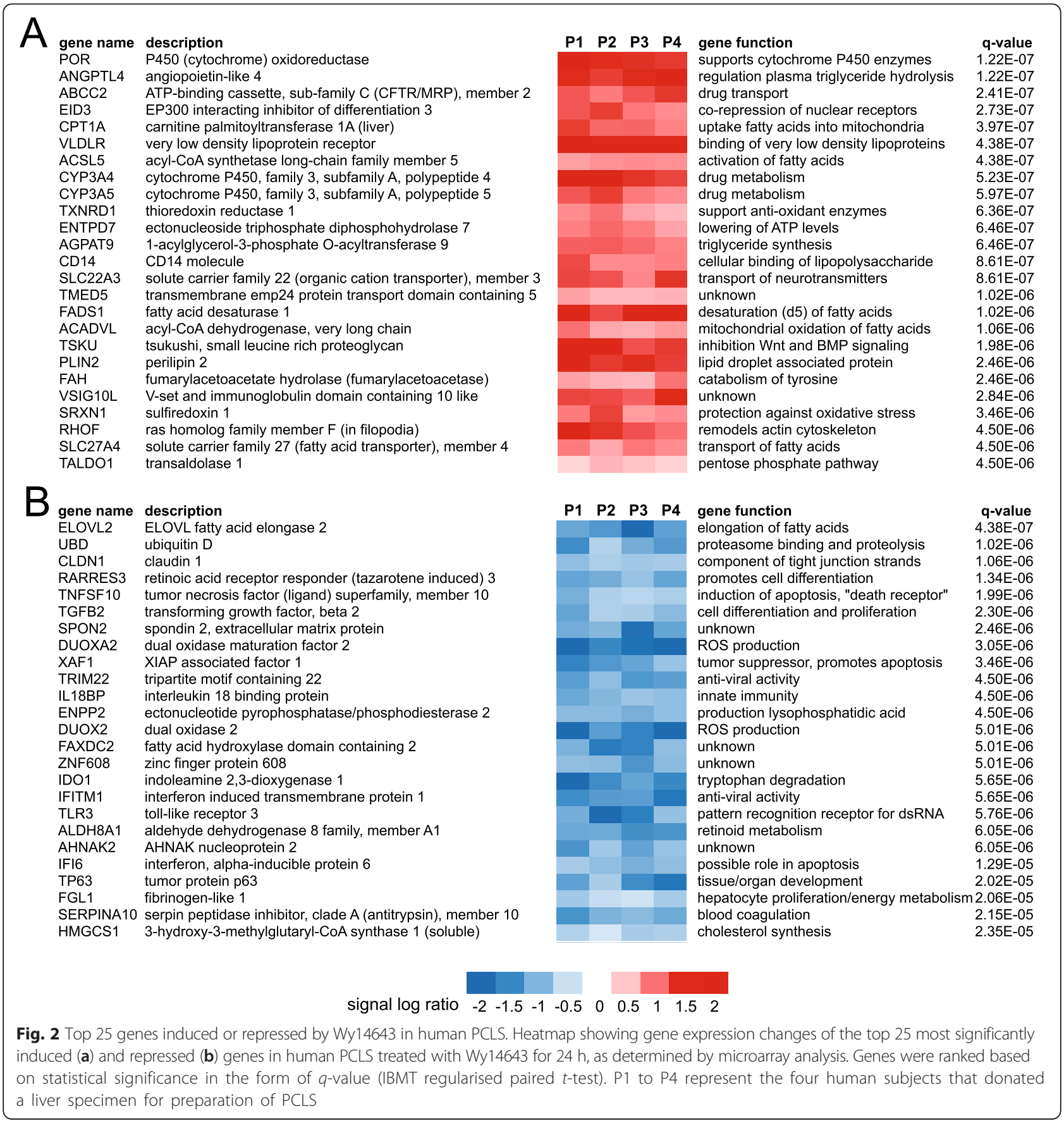


slices, we performed gene set enrichment analysis (GSEA). Pathways related to lipid metabolism or directly involving PPAR $\alpha$ featured prominently among the gene sets induced by Wy14643 (Fig. 3). Also, several gene sets induced by Wy14643 were related to the unfolded protein response and UPR signaling by IRE1 $\alpha$ and XBP1. Finally, we observed significant enrichment of genes related to oxidative stress and xenobiotic/drug metabolism. Gene sets downregulated by PPAR $\alpha$ activation were all related to immune function and inflammation, illustrating a potent anti-inflammatory/immuno-suppressive action of PPAR $\alpha$ in human liver.

An important goal of the present work was to test the suitability of liver slices as a model to study PPAR $\alpha$ dependent gene regulation and compare it with other available model systems for human liver. To that end, we compared the whole genome expression profiles of human liver slices treated with Wy14643 with the expression profiles of primary human hepatocytes treated with Wy14643. The expression profiles of primary human hepatocytes have been previously published but were re-analyzed [26]. The primary human hepatocytes were treated for the same duration and microarray analysis was performed in the same laboratory on the same microarray platform.

The comparative analysis between primary human hepatocytes and human liver slices was performed on a common gene set of 17,351 genes. Using a statistical cut-off of $P<0.001$ (IBMT regularised paired $t$-test) combined with a fold-change cut-off of 1.2 , we found 347 genes to be upregulated in human liver slices, as compared to 121 genes in the hepatocytes (Fig. 4a), with an overlap of 47 genes. Remarkably, whereas 401 genes were downregulated by Wy14643 in the liver slices, only 25 genes were downregulated by Wy14643 in the human hepatocytes, with an overlap of only 4 genes. The data

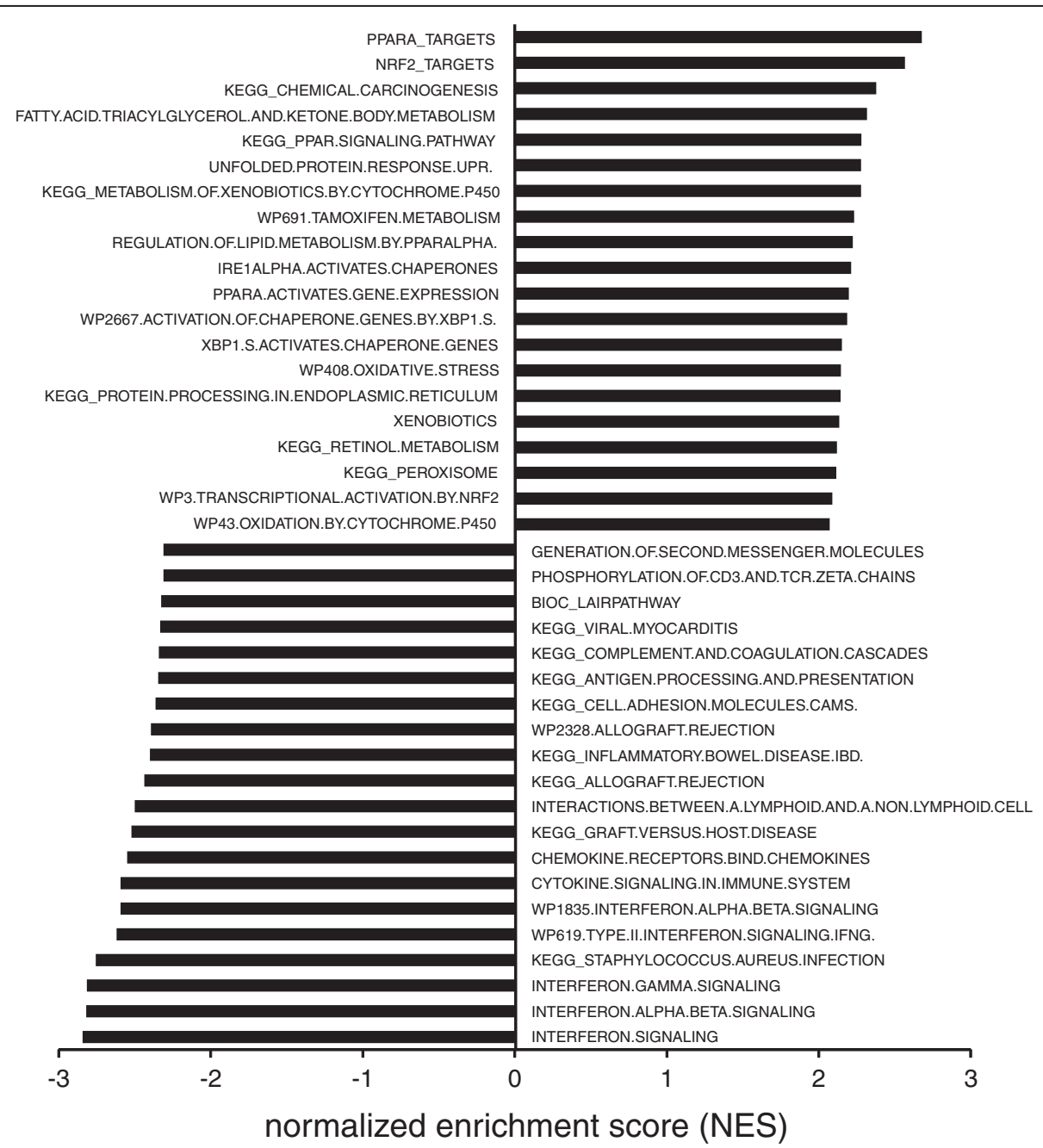

Fig. 3 Top 20 gene sets induced or repressed by Wy14643 in human PCLS. The top 20 most significantly induced or repressed pathways in human PCLS in response to $24 \mathrm{~h}$ Wy14643 treatment were determined by gene set enrichment analysis. Gene sets were ranked according to normalized enrichment score (NES) 


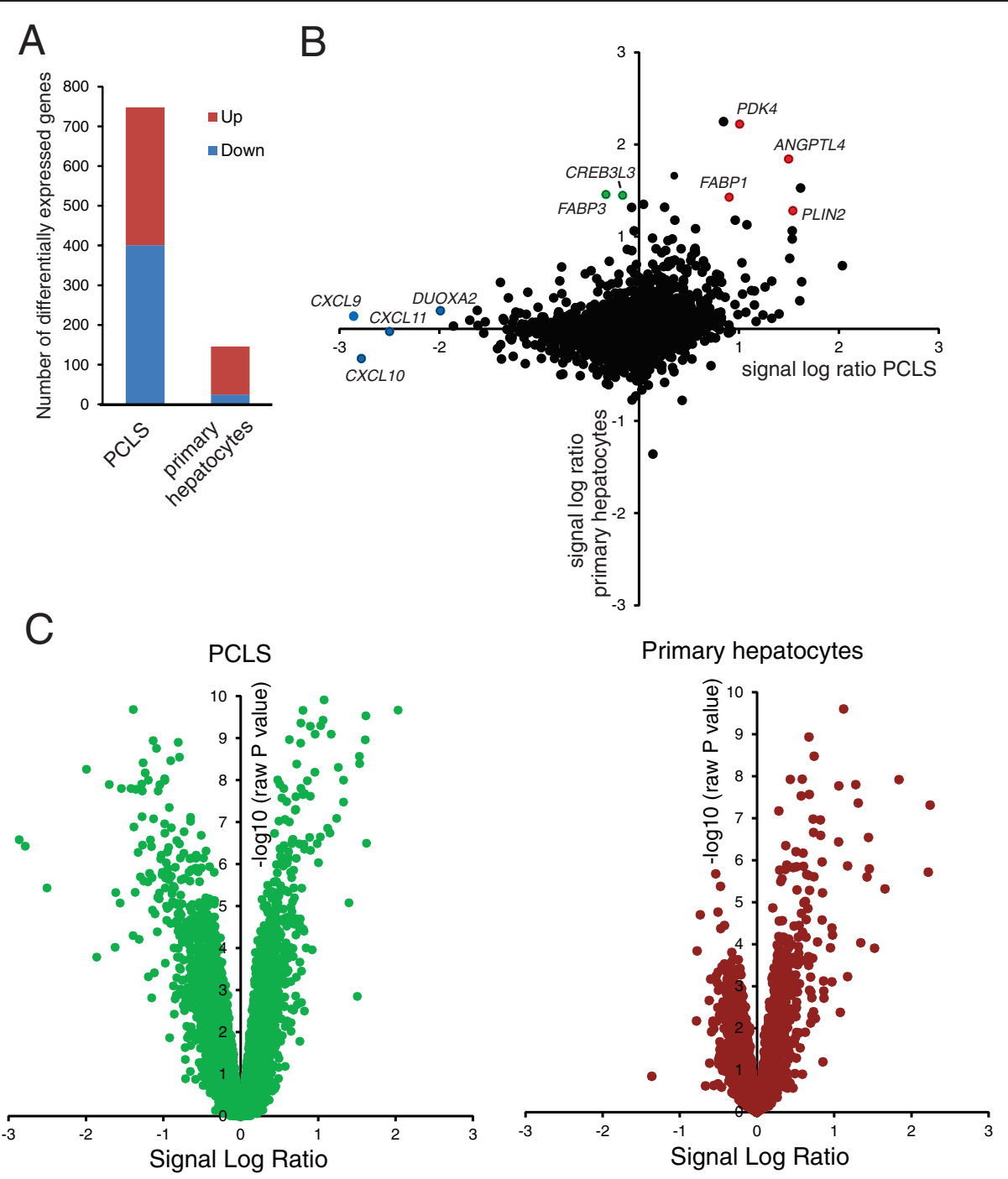

Fig. 4 Comparative analysis of effect Wy 14643 on gene expression in human PCLS and primary hepatocytes. a The number of differentially expressed genes in human PCLS and human primary hepatocytes in response to $24 \mathrm{~h}$ Wy14643 treatment in a common geneset of 17,351 genes was calculated based on a statistical significance cut-off of $P<0.001$ (IBMT regularised paired $t$-test) and fold-change $>1.20$. Genes were separated according to up- or down-regulation. $\mathbf{b}$ Correlation plot showing changes in gene expression in response to Wy14643 (expressed as signal log ratio) in human PCLS ( $\mathrm{x}$-axis) and primary human hepatocytes (y-axis). Selected PPARa target genes commonly induced by Wy14643 in PCLS and hepatocytes are highlighted in red. Selected inflammation-related genes specifically repressed by Wy14643 in PCLS are highlighted in blue. Selected lipid metabolism-related genes specifically induced by Wy14643 in primary hepatocytes are highlighted in green. c Volcano plot showing relative changes in gene expression in response to Wy14643 (expressed as signal log ratio, $x$-axis) plotted against statistical significance (expressed as IBMT regularised paired $t$-test $P$-value, $y$-axis) for the PCLS and primary hepatocytes

indicate that the human liver slices are much more sensitive towards especially downregulation of gene expression by Wy14643 as compared to human hepatocytes. This notion was further supported by correlation analysis in the form of a scatter plot (Fig. 4b). The upper right portion of the scatter plot was well filled (Fig. 4b), reflecting common induction by Wy14643 in PCLS and primary hepatocytes, including well-known PPAR $\alpha$ targets such as FABP1, PLIN2, PDK4 and ANGPTL4. In contrast, the lower left portion of the scatter plot was much less filled (Fig. 4b), reflecting little agreement between PCLS and primary hepatocytes with respect to downregulation of gene expression by Wy14643. In fact, many genes were markedly downregulated by Wy14643 in the liver slices but showed no change in expression in the hepatocytes. Nearly all genes conforming to this type of expression were involved in immune-related function, as illustrated by the chemokines CXCL9, CXCL10, and CXCL11. Interestingly, a number of genes was explicitly induced by PPAR $\alpha$ activation in primary hepatocytes but not in liver slices, including FABP3 as well as CREB3L3, a possible mediator of the stimulatory effects of PPAR $\alpha$ 
on hepatic gene expression [11]. Subsequent analysis by Volcano plot confirmed the overall more pronounced effect of PPAR $\alpha$ activation on gene expression in liver slices as compared to primary hepatocytes and also corroborated the relatively minor down-regulation of gene expression by PPAR $\alpha$ activation in primary hepatocytes (Fig. 4c).

To further investigate this observation, we took the 40 genes most highly induced or repressed by Wy14643 in the liver slices $(P<0.001$ and ranked according to fold-change) and studied the response to Wy14643 of the same genes in the primary hepatocytes. Whereas the majority of genes induced by Wy14643 in the liver slices were also induced by Wy14643 in the hepatocytes (Fig. 5a) - as illustrated by the common induction of well-known targets such as FABP1, PLIN2, FADS1 and ANGPTL4-few genes that were downregulated by Wy14643 in liver slices were also consistently downregulated by Wy14643 in primary hepatocytes (Fig. 5b). Also, the magnitude of gene suppression by Wy14643 was generally much less pronounced in primary hepatocytes. Confirming the GSEA results, the majority of the most highly repressed genes were related to immunity and inflammation, including the aforementioned chemokines (CXCL9-11, CCL8, CX3CL1, CXCL6), interferon $\gamma$-induced genes (IFITM1, IFIT1, IFIT2, IFIT3) and other immune-related genes (TLR3, NOS2, and LCN2).

To further explore the similarity in gene regulation by Wy14643 between PCLS and primary hepatocytes, we

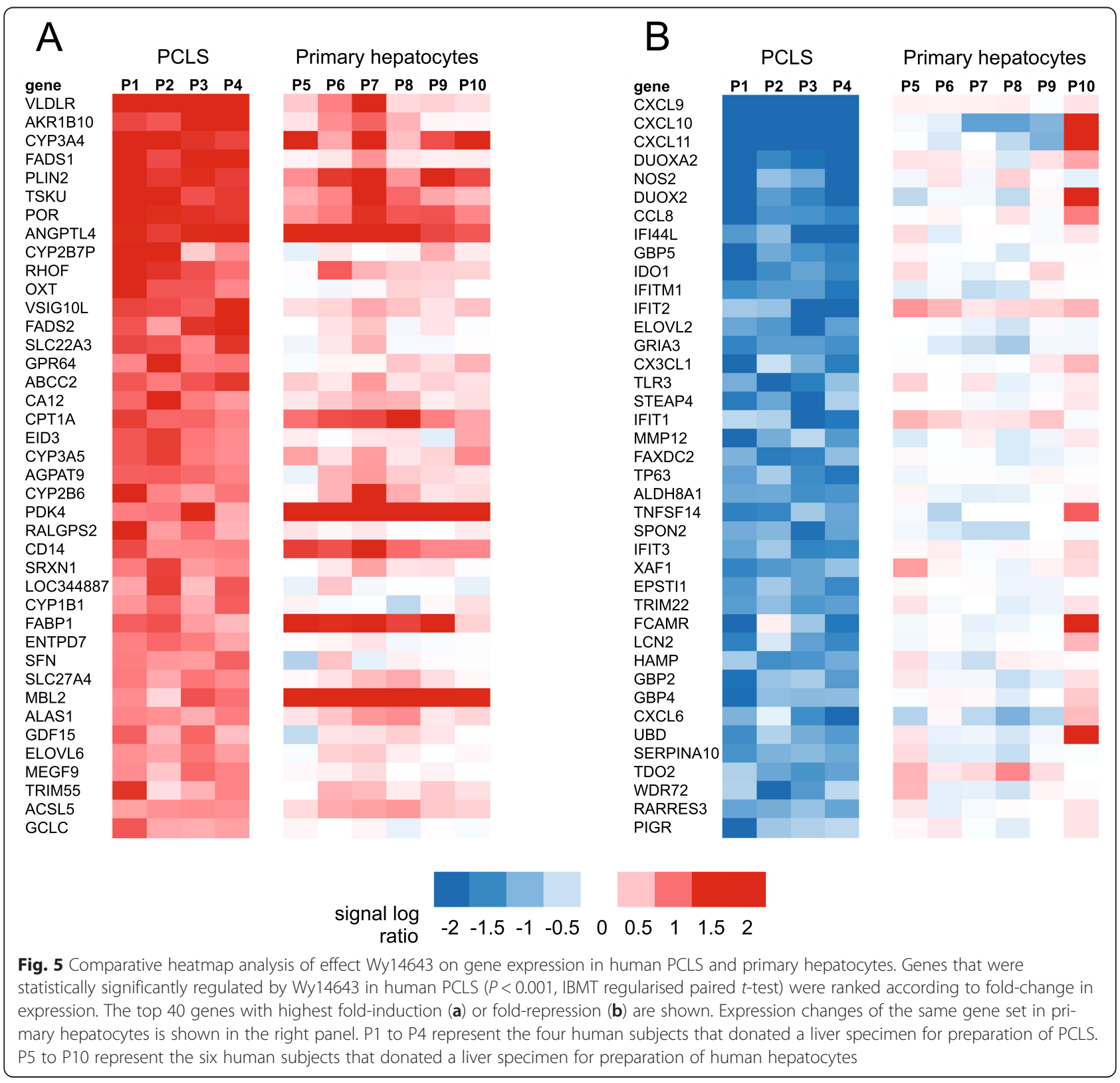


took the enriched genes within the positively or negatively enriched gene sets "IRE1 $\alpha$ activated chaperones", "metabolism of xenobiotics by cytochrome P450", and "Interferon alpha beta signaling" (Fig. 3), and compared gene expression changes between PCLS and primary hepatocytes. Induction of most genes that are part of "metabolism of xenobiotics by cytochrome P450" was more pronounced in PCLS than in primary hepatocytes but reasonably well conserved between the two model systems (Fig. 6a). A limited number of genes (i.e. CYP2J2) showed higher fold-inductions in the primary hepatocytes as compared to PCLS. Induction of genes part of "IRE1 $\alpha$ activated chaperones" was generally less pronounced in comparison with genes part of "metabolism of xenobiotics by cytochrome P450", and was relatively weakly conserved between PCLS and primary hepatocytes (Fig. 6b). An exception is ACADVL. However, the inclusion of ACADVL (very long chain acyl-CoA dehydrogenase $=$ fatty acid oxidation) within "IRE1 $\alpha$ activated chaperones" may be questioned. Consistent with the other data showing potent downregulation of immune- and inflammation-related genes by PPAR $\alpha$ activation in PCLS but not primary hepatocytes, suppression of genes part of "Interferon alpha beta signaling" was exclusively observed in PCLS (Fig. 6c).

Finally, we used the microarray data of the human primary hepatocytes and human PCLS treated with Wy14643 to generate a detailed gene map of known and putative PPAR $\alpha$ target genes involved in lipid metabolic pathways (Fig. 7). The map illustrates that regulation of bile acid synthesis and secretion, which is a well-established PPAR $\alpha$ target pathway in mouse, was only evident in primary hepatocytes and not in liver slices. Conversely, genes involved in fatty acid elongation and desaturation were clearly induced by PPAR $\alpha$ activation in human liver slices but not in primary hepatocytes.

\section{Discussion}

The main conclusion of our study is that induction of gene expression by PPAR $\alpha$ activation is generally well captured and shows significant overlap between human liver slices and primary human hepatocytes, showing consistent upregulation of genes involved in lipid and xenobiotic metabolism in the two model systems. In contrast, downregulation of gene expression by PPAR $\alpha$ activation is almost exclusively observed in human liver slices. A previous study comparing mouse primary hepatocytes, mouse liver slices and mouse liver reached a similar conclusion [30]. Overall, our data indicate that human PCLS are a superior model to study PPAR $\alpha-$ dependent gene regulation and PPAR $\alpha$ functions in human liver.

As indicated above, PPAR $\alpha$ activation caused major downregulation of gene expression in human liver slices but not in primary hepatocytes. A key difference between primary hepatocytes and liver slices is that the primary hepatocyte culture consists of only hepatocytes, whereas the liver slices contain other cell types, including stellate cells and Kupffer cells. A large portion of the downregulated genes and pathways in the liver slices was found to be connected to the immune system. Genes that were highly repressed upon PPAR $\alpha$ activation included several chemokines (e.g. CXCL9-11, CCL8, CX3CL1, CXCL6), interferon $\gamma$-induced genes (e.g. IFITM1, IFIT1, IFIT2, IFIT3) and numerous other immune-related genes (e.g. TLR3, NOS2, and LCN2). Downregulation of gene expression is unlikely to be mediated by PPAR $\alpha$ present in nonparenchymal cells, as PPAR $\alpha$ expression in these cells is very low [30, 39]. Instead we prefer a scenario in which the immuno-suppressive action of PPAR $\alpha$ activation in hepatocytes is dependent on (inflammatory) signals emanating from non-parenchymal cells. Indeed, downregulation of inflammatory gene expression in primary hepatocytes and mouse liver by PPAR $\alpha$ activation is sensitive to the presence of pro-inflammatory stimuli $[40,41]$. Previously, we and others already demonstrated that Kupffer cells promote fat storage in hepatocytes by releasing inflammatory signals such as IL-1 $\beta$, causing downregulation of PPAR $\alpha$ gene expression [42, 43]. Overall, these data suggest that the full scope of functions of PPAR $\alpha$ in hepatocytes is critically dependent on the interaction with other liver cell types. It is of interest to note that recently the anti-inflammatory action of PPAR $\alpha$ in mouse liver was unequivocally attributed to the ability of PPAR $\alpha$ to interact with other transcription factor pathways-a property referred to as transrepression-independent of the DNA-binding ability of PPAR $\alpha$, as shown in the context of steatohepatitis [44].

Despite the clear clinical efficacy of fibrates towards lowering of circulating triglycerides, the lack of peroxisome proliferation in human primary hepatocytes following PPAR $\alpha$ activation has fed the idea that humans are largely insensitive to peroxisome-proliferatorinduced hepatic effects and that the functional role of PPAR $\alpha$ in human liver may be limited [45-47]. Subsequent whole genome expression profiling studies in primary human hepatocytes have mostly discounted these notions $[25,26]$. We found that PPAR $\alpha$ is highly expressed in human liver with $\mathrm{Ct}$ values ranging from 22 to 26 , which is similar to the values observed in mouse liver (data not shown). Importantly, despite the markedly lower expression of PPAR $\alpha$ in human PCLS as compared to human liver, activation of PPAR $\alpha$ in PCLS caused pronounced upregulation or downregulation of numerous genes, including many known PPAR $\alpha$ targets, strongly supporting the functionality of PPAR $\alpha$ in human PCLS and giving strong credibility to an important in vivo role of PPAR $\alpha$ in human liver. Recently, it was found that human liver PPAR $\alpha$ gene expression correlates negatively 


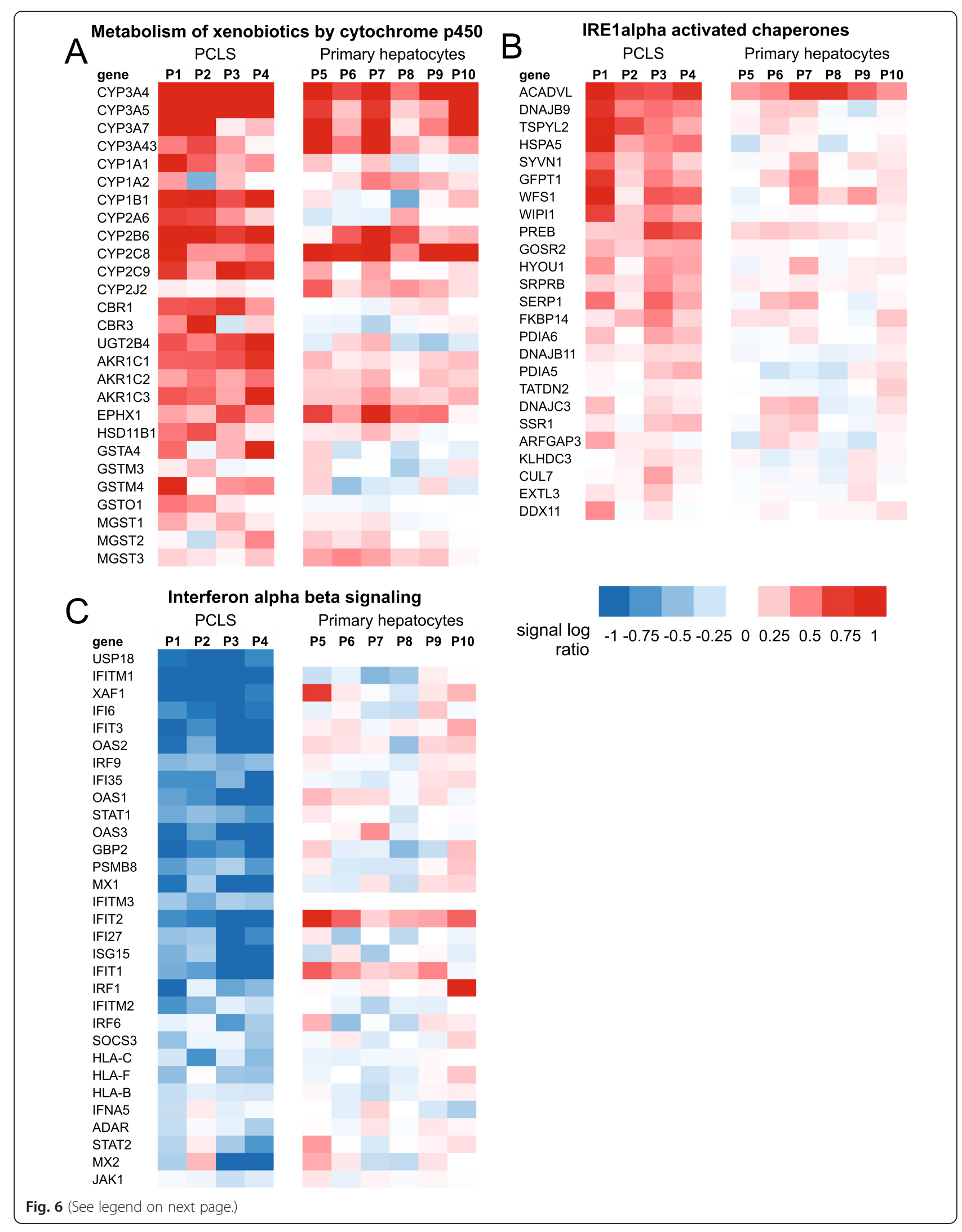


(See figure on previous page.)

Fig. 6 Regulation of selected gene sets by Wy14643 in human PCLS and primary hepatocytes. Heatmap showing gene expression changes of enriched genes that are part of the gene sets "metabolism of xenobiotics by cytochrome P450" (a), "IRE1a activated chaperones" (b), and "Interferon alpha beta signaling" (c) in human PCLS and primary hepatocytes. P1 to P4 represent the four human subjects that donated a liver specimen for preparation of PCLS. P5 to P10 represent the six human subjects that donated a liver specimen for preparation of human hepatocytes

with the severity of steatohepatitis and with measures of insulin resistance. Furthermore, histological improvement in a follow-up biopsy was associated with increased expression of PPAR $\alpha$ and its target genes, suggesting that PPAR $\alpha$ is involved in and may be therapeutically targeted for human steatohepatitis [48].

The majority of gene sets enriched among the upregulated genes were related to lipid and xenobiotic metabolism, which are well-established target pathways of PPAR $\alpha$. Intriguingly, several highly enriched gene sets were part of the unfolded protein response and IRE1 $\alpha$ XBP1 signaling, two key factors involved in governing UPR. Currently, there are no published data linking PPAR $\alpha$ to IRE1 $\alpha-X B P 1$ signaling and regulation of UPR, though it has been observed that PPAR $\alpha$ is involved in regulating proteome maintenance by inducing numerous heat shock proteins [49]. Surprisingly, recently it was demonstrated that IRE1 $\alpha-\mathrm{XBP} 1$ signaling leads to activation of PPAR $\alpha$ via direct binding of XBP1s to the promoter of PPAR $\alpha$, thereby stimulating mitochondrial $\beta$ oxidation and ketogenesis [50]. Thus, there appear to be reciprocal interactions between PPAR $\alpha$ and UPR. The wider biological framework for regulation of UPR requires further clarification.

Our analysis reveals differential regulation of a number of PPAR $\alpha$ targets between liver slices and primary hepatocytes. For instance, VNN1 was significantly upregulated by PPAR $\alpha$ activation in primary hepatocytes but

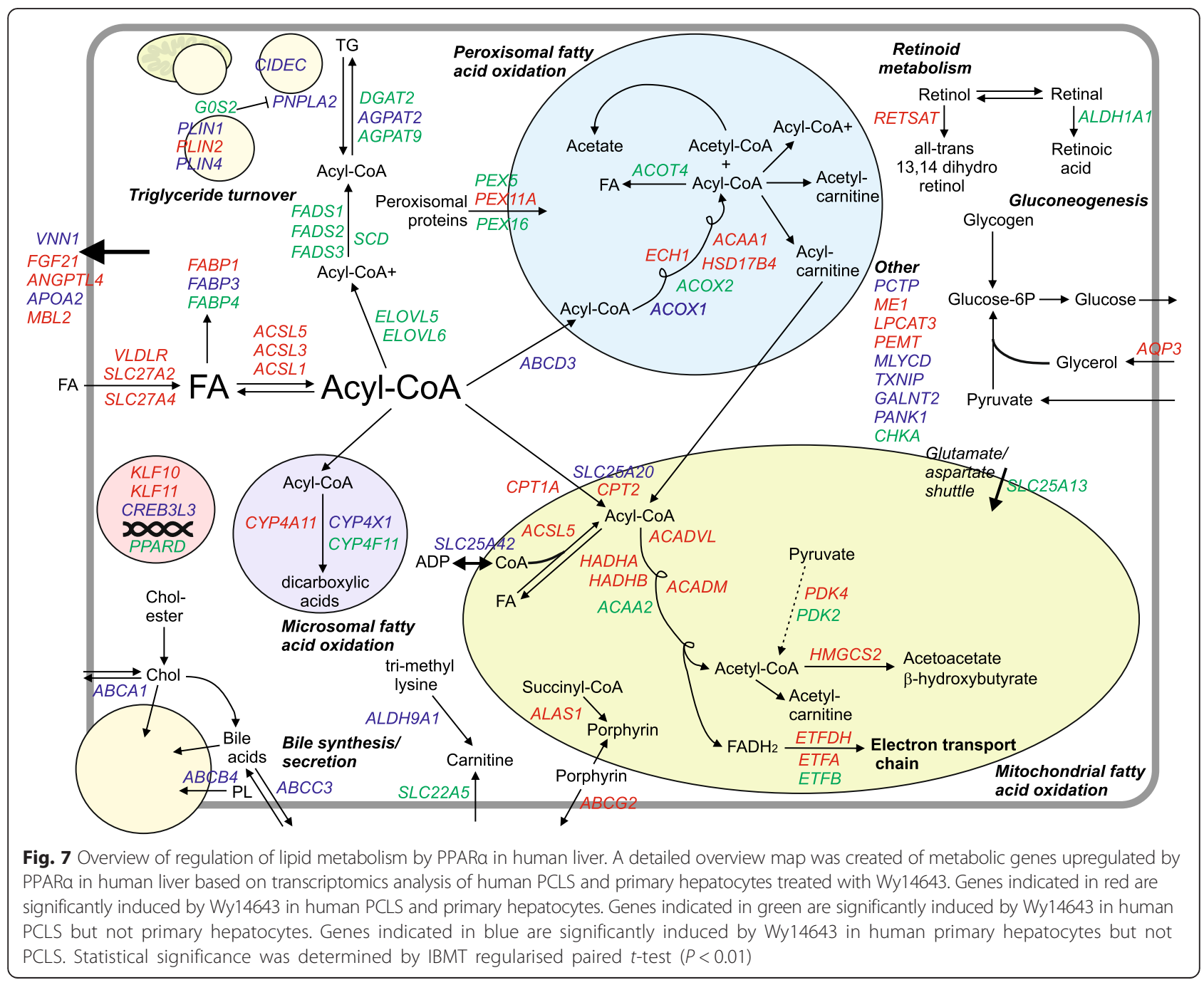


showed no change in expression in liver slices. Conversely, expression of FADS2 was significantly increased in human liver slices but showed no change in primary hepatocytes. The differential regulation of specific PPAR $\alpha$ target genes by Wy14643 between primary hepatocytes and liver slices may be a reflection of the different cellular context in the two models systems, with non-hepatocytes potentially exerting a stimulatory or inhibitory influence on PPAR $\alpha$ dependent gene induction. However, it should be realized that for a number of genes the seemingly differential regulation may reflect a quantitative difference rather than a true qualitative difference. For example, SLC25A20 was induced significantly by 1.58 -fold in primary hepatocytes as compared to a non-significant 1.34-fold induction in liver slices, barely missing the statistical significance cut-off.

Our analysis yielded a number of relatively poorly characterized genes that showed a pronounced and consistent upregulation upon PPAR $\alpha$ activation in the two human liver model systems. These include TSKU, RHOF, CA12 and VSIG10L. Interestingly, many genes that were found to be induced by PPAR $\alpha$ in early microarray analyses and which did not have an assigned function at the time were later shown to be involved in some aspect of lipid metabolism. Accordingly, it can be hypothesized that the above mentioned genes as well as other poorly characterized genes that are commonly induced by Wy14643 in various liver model systems may be directly or indirectly connected to lipid metabolism.

The comparative microarray analysis of the effect of PPAR $\alpha$ activation in primary human hepatocytes and human liver slices is somewhat hampered by a number of different factors, including the use of different types of Affymetrix gene chips, different human donors, and an unequal number of biological replicates per group. However, treatments of liver slices and hepatocytes were carried out for the same duration and with the same PPAR $\alpha$ agonist. Furthermore, RNA was isolated and labeled via the same technique, hybridizations were performed on the same platform by the same technician, and the microarray data were processed in parallel using the same analysis methods.

On a final note, the data collected in this paper were added to a publicly available overview map of known (lipid) metabolic genes upregulated by PPAR $\alpha$ in human liver (accessible via: http://en.wikipedia.org/wiki/Peroxisome_proliferator-activated_receptor_alpha), which was generated largely by using published transcriptome datasets.

\section{Conclusion}

In conclusion, our paper demonstrates the suitability and superiority of PCLS over primary human hepatocytes for studying the functional role of PPAR $\alpha$ in human liver. Our data underscore the major role of PPAR $\alpha$ in regulation of hepatic lipid and xenobiotic metabolism and reveal a marked immuno-suppressive/antiinflammatory effect of PPAR $\alpha$ in human liver that may be therapeutically relevant for NAFLD. The data add to our growing understanding of the critical role of PPAR $\alpha$ in gene regulation in human liver.

\section{Additional files}

\section{Additional file 1: Full list of significantly induced genes by Wy14643 in human PCLS. (PDF $211 \mathrm{~kb}$ ) \\ Additional file 2: Full list of significantly repressed genes by Wy14643 in human PCLS. (PDF 228 kb)}

\section{Abbreviations}

PPARa: Peroxisome proliferator activated receptor alpha; PCLS: Precision cut liver slices; NAFLD: Non-alcoholic fatty liver disease; DMSO: Dimethyl sulfoxide; GSEA: Gene set enrichment analysis; IBMT: Intensity-Based Moderated T-statistic.

\section{Competing interests}

The authors declare that they have no competing interests.

\section{Authors' contributions}

AJ designed the study, performed experiments, analyzed data and edited the manuscript. BB designed the study and edited the manuscript. GS performed experiments. FB designed the study and collected samples. IJ designed the study and collected samples. AP designed the study and edited the manuscript. SK designed the study, analyzed data, and wrote the manuscript. All authors approved the final manuscript.

\section{Acknowledgements}

This research was supported by CVON IN-CONTROL grant CVON2012-03.

\section{Author details}

${ }^{1}$ Nutrition, Metabolism and Genomics Group, Wageningen University, Stippeneng 4, 6708 WE, Wageningen, The Netherlands. 'Department of Surgery, Rijnstate Hospital, Wagnerlaan 55, 6815 AD, Arnhem, The Netherlands. ${ }^{3}$ RIKILT_Institute of Food Safety, Wageningen UR, P.O. Box 230, 6700 AE, Wageningen, The Netherlands.

Received: 31 March 2015 Accepted: 30 September 2015

Published online: 08 October 2015

\section{References}

1. Kersten S, Desvergne B, Wahli W. Roles of PPARs in health and disease. Nature. 2000;405(6785):421-4.

2. Dreyer C, Krey G, Keller H, Givel F, Helftenbein G, Wahli W. Control of the peroxisomal beta-oxidation pathway by a novel family of nuclear hormone receptors. Cell. 1992;68(5):879-87.

3. Braissant O, Foufelle F, Scotto C, Dauca M, Wahli W. Differential expression of peroxisome proliferator-activated receptors (PPARs): tissue distribution of PPAR-alpha, -beta, and-gamma in the adult rat. Endocrinology. 1996;137(1):354-66.

4. Wagner KD, Wagner N. Peroxisome proliferator-activated receptor beta/ delta (PPARbeta/delta) acts as regulator of metabolism linked to multiple cellular functions. Pharmacol Ther. 2010;125(3):423-35.

5. Tontonoz P, Hu E, Spiegelman BM. Stimulation of adipogenesis in fibroblasts by PPAR gamma 2, a lipid-activated transcription factor. Cell. 1994;79(7):1147-56.

6. Tontonoz P, Spiegelman BM. Fat and beyond: the diverse biology of PPARgamma. Annu Rev Biochem. 2008;77:289-312.

7. Lehmann JM, Moore LB, Smith-Oliver TA, Wilkison WO, Willson TM, Kliewer SA. An antidiabetic thiazolidinedione is a high affinity ligand for peroxisome proliferator-activated receptor gamma (PPAR gamma). J Biol Chem. 1995;270(22):12953-6.

8. Hashimoto T, Cook WS, Qi C, Yeldandi AV, Reddy JK, Rao MS. Defect in peroxisome proliferator-activated receptor alpha-inducible fatty acid 
oxidation determines the severity of hepatic steatosis in response to fasting. J Biol Chem. 2000;275(37):28918-28.

9. Kersten S, Seydoux J, Peters JM, Gonzalez FJ, Desvergne B, Wahli W. Peroxisome proliferator-activated receptor alpha mediates the adaptive response to fasting. J Clin Invest. 1999;103(11):1489-98.

10. Leone TC, Weinheimer CJ, Kelly DP. A critical role for the peroxisome proliferator-activated receptor alpha (PPARalpha) in the cellular fasting response: the PPARalpha-null mouse as a model of fatty acid oxidation disorders. Proc Natl Acad Sci U S A. 1999;96(13):7473-8.

11. Kersten S. Integrated physiology and systems biology of PPARalpha. Mol Metab. 2014;3(4):354-71.

12. Dreyer C, Keller H, Mahfoudi A, Laudet V, Krey G, Wahli W. Positive regulation of the peroxisomal beta-oxidation pathway by fatty acids through activation of peroxisome proliferator-activated receptors (PPAR). Biol Cell Eur Cell Biol Organ. 1993;77(1):67-76.

13. Forman BM, Chen J, Evans RM. Hypolipidemic drugs, polyunsaturated fatty acids, and eicosanoids are ligands for peroxisome proliferator-activated receptors alpha and delta. Proc Natl Acad Sci U S A. 1997;94(9):4312-7.

14. Gottlicher M, Widmark E, Li Q, Gustafsson JA. Fatty acids activate a chimera of the clofibric acid-activated receptor and the glucocorticoid receptor. Proc Natl Acad Sci U S A. 1992;89(10):4653-7.

15. Kliewer SA, Sundseth SS, Jones SA, Brown PJ, Wisely GB, Koble CS, et al. Fatty acids and eicosanoids regulate gene expression through direct interactions with peroxisome proliferator-activated receptors alpha and gamma. Proc Natl Acad Sci U S A. 1997;94(9):4318-23.

16. Krey G, Braissant O, L'Horset F, Kalkhoven E, Perroud M, Parker MG, et al. Fatty acids, eicosanoids, and hypolipidemic agents identified as ligands of peroxisome proliferator-activated receptors by coactivator-dependent receptor ligand assay. Mol Endocrinol. 1997;11(6):779-91.

17. Issemann I, Green S. Activation of a member of the steroid hormone receptor superfamily by peroxisome proliferators. Nature. 1990;347(6294):645-50.

18. Staels B, Fruchart JC. Therapeutic roles of peroxisome proliferator-activated receptor agonists. Diabetes. 2005;54(8):2460-70.

19. Staels B, Rubenstrunk A, Noel B, Rigou G, Delataille P, Millatt $\sqcup$, et al. Hepatoprotective effects of the dual peroxisome proliferator-activated receptor alpha/delta agonist, GFT505, in rodent models of nonalcoholic fatty liver disease/nonalcoholic steatohepatitis. Hepatology. 2013;58(6):1941-52.

20. Cariou B, Hanf R, Lambert-Porcheron S, Zair Y, Sauvinet V, Noel B, et al. Dual peroxisome proliferator-activated receptor alpha/delta agonist GFT505 improves hepatic and peripheral insulin sensitivity in abdominally obese subjects. Diabetes Care. 2013;36(10):2923-30.

21. Lee SS, Pineau T, Drago J, Lee EJ, Owens JW, Kroetz DL, et al. Targeted disruption of the alpha isoform of the peroxisome proliferator-activated receptor gene in mice results in abolishment of the pleiotropic effects of peroxisome proliferators. Mol Cell Biol. 1995;15(6):3012-22.

22. Corton JC, Cunningham ML, Hummer BT, Lau C, Meek B, Peters JM, et al Mode of action framework analysis for receptor-mediated toxicity: The peroxisome proliferator-activated receptor alpha (PPARalpha) as a case study. Crit Rev Toxicol. 2014;44(1):1-49.

23. Blaauboer BJ, van Holsteijn CW, Bleumink R, Mennes WC, van Pelt FN, Yap $\mathrm{SH}$, et al. The effect of beclobric acid and clofibric acid on peroxisomal beta-oxidation and peroxisome proliferation in primary cultures of rat, monkey and human hepatocytes. Biochem Pharmacol. 1990;40(3):521-8.

24. Cattley RC, DeLuca J, Elcombe C, Fenner-Crisp P, Lake BG, Marsman DS, et al. Do peroxisome proliferating compounds pose a hepatocarcinogenic hazard to humans? Regul Toxicol Pharmacol. 1998;27(1 Pt 1):47-60.

25. McMullen PD, Bhattacharya S, Woods CG, Sun B, Yarborough K, Ross SM, et al. A map of the PPARalpha transcription regulatory network for primary human hepatocytes. Chem Biol Interact. 2013;209C:14-24.

26. Rakhshandehroo M, Hooiveld G, Muller M, Kersten S. Comparative analysis of gene regulation by the transcription factor PPARalpha between mouse and human. PLoS One. 2009:4(8):e6796.

27. Smith PF, Gandolfi AJ, Krumdieck CL, Putnam CW, Zukoski 3rd CF, Davis WM, et al. Dynamic organ culture of precision liver slices for in vitro toxicology. Life Sci. 1985;36(14):1367-75

28. Jung D, Elferink MG, Stellaard F, Groothuis GM. Analysis of bile acid-induced regulation of FXR target genes in human liver slices. Liver Int. 2007;27(1):137-44.

29. Mattijssen F, Georgiadi A, Andasarie T, Szalowska E, Zota A, Krones-Herzig A, et al. Hypoxia-inducible lipid droplet-associated (HILPDA) is a novel peroxisome proliferator-activated receptor (PPAR) target involved in hepatic triglyceride secretion. J Biol Chem. 2014;289(28):19279-93.
30. Szalowska E, Tesfay HA, van Hijum SA, Kersten S. Transcriptomic signatures of peroxisome proliferator-activated receptor alpha (PPARalpha) in different mouse liver models identify novel aspects of its biology. BMC Genomics. 2014;15:1106.

31. Szalowska E, van der Burg B, Man HY, Hendriksen PJ, Peijnenburg AA. Model steatogenic compounds (amiodarone, valproic acid, and tetracycline) alter lipid metabolism by different mechanisms in mouse liver slices. PLoS One. 2014;9(1):e86795.

32. Willson TM, Brown PJ, Sternbach DD, Henke BR. The PPARs: from orphan receptors to drug discovery. J Med Chem. 2000;43(4):527-50.

33. Bolstad BM, Irizarry RA, Astrand M, Speed TP. A comparison of normalization methods for high density oligonucleotide array data based on variance and bias. Bioinformatics. 2003:19(2):185-93.

34. Irizarry RA, Bolstad BM, Collin F, Cope LM, Hobbs B, Speed TP. Summaries of Affymetrix GeneChip probe level data. Nucleic Acids Res. 2003;31(4):e15.

35. Dai M, Wang P, Boyd AD, Kostov G, Athey B, Jones EG, et al. Evolving gene/ transcript definitions significantly alter the interpretation of GeneChip data. Nucleic Acids Res. 2005;33(20):e175.

36. Sartor MA, Tomlinson CR, Wesselkamper SC, Sivaganesan S, Leikauf GD, Medvedovic M. Intensity-based hierarchical Bayes method improves testing for differentially expressed genes in microarray experiments. BMC Bioinf. 2006;7:538.

37. Storey JD, Tibshirani R. Statistical significance for genomewide studies. Proc Natl Acad Sci U S A. 2003;100(16):9440-5.

38. Subramanian A, Tamayo P, Mootha VK, Mukherjee S, Ebert BL, Gillette MA et al. Gene set enrichment analysis: a knowledge-based approach for interpreting genome-wide expression profiles. Proc Natl Acad Sci U S A. 2005;102(43):15545-50.

39. Peters JM, Rusyn I, Rose ML, Gonzalez FJ. Thurman RG: peroxisome proliferatoractivated receptor alpha is restricted to hepatic parenchymal cells, not Kupffer cells: implications for the mechanism of action of peroxisome proliferators in hepatocarcinogenesis. Carcinogenesis. 2000;21(4):823-6.

40. Gervois P, Kleemann R, Pilon A, Percevault F, Koenig W, Staels B, et al. Global suppression of IL-6-induced acute phase response gene expression after chronic in vivo treatment with the peroxisome proliferator-activated receptor-alpha activator fenofibrate. J Biol Chem. 2004:279(16):16154-60.

41. Gervois P, Vu-Dac N, Kleemann R, Kockx M, Dubois G, Laine B, et al. Negative regulation of human fibrinogen gene expression by peroxisome proliferator-activated receptor alpha agonists via inhibition of CCAAT box/ enhancer-binding protein beta. J Biol Chem. 2001;276(36):33471-7.

42. Fang X, Zou S, Zhao Y, Cui R, Zhang W, Hu J, et al. Kupffer cells suppress perfluorononanoic acid-induced hepatic peroxisome proliferator-activated receptor alpha expression by releasing cytokines. Arch Toxicol. 2012;86(10):1515-25.

43. Stienstra R, Saudale F, Duval C, Keshtkar S, Groener JE, van Rooijen N, et al. Kupffer cells promote hepatic steatosis via interleukin-1beta-dependent suppression of peroxisome proliferator-activated receptor alpha activity. Hepatology. 2010;51(2):511-22.

44. Pawlak M, Bauge E, Bourguet W, De Bosscher K, Lalloyer F, Tailleux A, et al. The transrepressive activity of peroxisome proliferator-activated receptor alpha is necessary and sufficient to prevent liver fibrosis in mice. Hepatology. 2014;60(5):1593-606

45. Bentley P, Calder I, Elcombe C, Grasso P, Stringer D, Wiegand HJ. Hepatic peroxisome proliferation in rodents and its significance for humans. Food Chem Toxicology. 1993;31(11):857-907.

46. Palmer CN, Hsu MH, Griffin KJ, Raucy JL, Johnson EF. Peroxisome proliferator activated receptor-alpha expression in human liver. Mol Pharmacol. 1998:53(1):14-22

47. Holden PR, Tugwood JD. Peroxisome proliferator-activated receptor alpha: role in rodent liver cancer and species differences. J Mol Endocrinol. 1999;22(1):1-8

48. Francque S, Verrijken A, Caron S, Prawitt J, Paumelle R, Derudas B, et al. PPARalpha gene expression correlates with severity and histological treatment response in patients with Non-alcoholic Steatohepatitis. J Hepatol. 2015;63(1):164-73.

49. Vallanat B, Anderson SP, Brown-Borg HM, Ren H, Kersten S, Jonnalagadda S, et al. Analysis of the heat shock response in mouse liver reveals transcriptional dependence on the nuclear receptor peroxisome proliferator-activated receptor alpha (PPARalpha). BMC Genomics. 2010;11:16.

50. Shao M, Shan B, Liu Y, Deng Y, Yan C, Wu Y, et al. Hepatic IRE1alpha regulates fasting-induced metabolic adaptive programs through the XBP1s-PPARalpha axis signalling. Nat Commun. 2014;5:3528. 